\title{
Novel Testing to Study the Performance of Intumescent Coatings under Non-Standard Heating Regimes
}

\author{
ANGUS ELLIOTT, ALISTAIR TEMPLE, CRISTIAN MALUK, and LUKE BISBY \\ School of Engineering \\ University of Edinburgh \\ The King's Buildings, Mayfield Road \\ Edinburgh, UK EH93JL
}

\begin{abstract}
Intumescent coatings (also called reactive coatings) are widely used to protect structural steel from fire. These thin coatings swell on heating to form a highly insulating char, protecting steel members and preventing them from reaching critical temperatures that could cause them to fail. As is the case for most structural materials and assemblies, intumescent coatings for use in buildings are typically developed and certified solely according to the standard cellulosic fire resistance test by exposure within a fire testing furnace. Reliance on furnace testing is expensive, non-representative of realistic fire conditions, and insufficiently versatile to gather detailed performance information on the response of reactive coatings under the full range of design fires which might be considered during a rational, performance-based design assessment. This paper presents a novel testing methodology for studying the performance of reactive coatings when subjected to non-standard heating regimes. The new approach is calibrated and validated using furnace test data, and is shown to offer considerable advantages over furnace testing in terms of reliability, repeatability, versatility, speed and cost. An investigation is then presented to study the effective variable thermal conductivity of a commercially available reactive coating when subjected to various timehistories of heat flux. It is shown that the heating rate and dry film thickness of the coating do not drastically affect the development of effective thermal conductivity with substrate temperature, leading to a proposal for a simplified method for specifying coating requirements and/or performing heat transfer design calculations when designing to non-standard heating regimes.
\end{abstract}

KEYWORDS: Protection of steel, performance-based design, heat transfer, structural design.

\section{NOMENCLATURE LISTING}

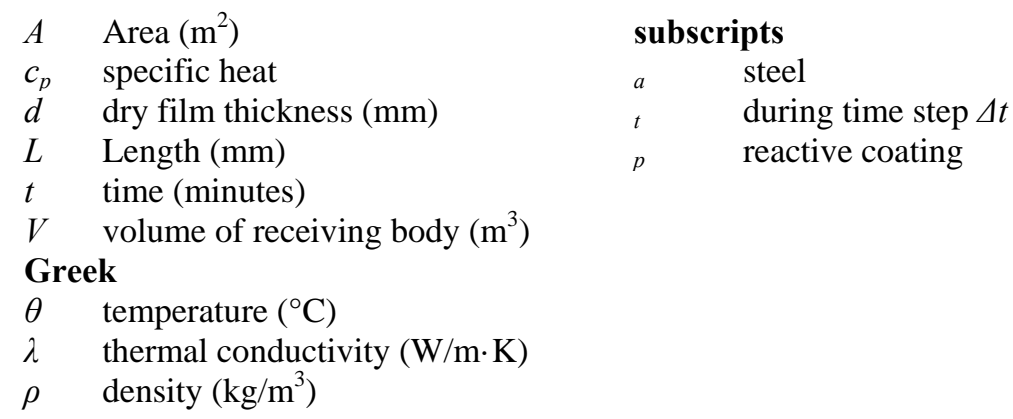

\section{INTRODUCTION}

In recent decades intumescent (reactive) coatings have found widespread use for the protection of structural steel from fire. In the event of a fire, heating causes the coating to expand and form a layer of thermally insulating char which delays heat transfer to the underlying substrate. Reactive coatings are typically designed to be applied at dry film thicknesses (DFTs) of only a few millimeters, but may expand to between 50 and 200 times their original thickness. They are increasingly popular in practice as they do not interfere with a structure's aesthetics and in many cases can be applied off site [1].

Reactive coatings used for fire protection of structural steel elements are currently tested for development and compliance in furnaces according to BS EN 1363-1 [2], or ASTM E119 [3] in North America. The test procedure to determine the required DFT involves subjecting a test specimen to a 'standard' temperaturetime curve in a furnace and measuring the time for the steel temperature to reach a 'critical temperature' 
(normally in the range of $550^{\circ} \mathrm{C}$ for reasons which are not discussed here). The standard temperature-time curve is intended to represent a worst-case building compartment fire assuming cellulosic fuel. While furnace tests are useful for comparing the relative performance of similar materials under a single severe heating condition, furnace tests cannot properly account for important factors such as the real fire and compartment conditions, loading, or failure modes [4]; thus inhibiting advances in understanding how reactive coatings behave when subjected to non-standard time-temperature curves such as those likely to occur in a real fire, or understanding the extent to which the rate of heating might affect the resulting char formation and fire protection.

There is also some question as to whether furnace tests genuinely allow for meaningful relative comparison between different materials [5]. Furnace testing was developed in the early $20^{\text {th }}$ Century to replicate a presumed 'worst case' fire at that time, yet it is still used for assessment of modern materials very different from their historical counterparts [6]. Furnace testing is, however, unsuitable for relative assessment of materials with significantly different thermal properties [7]. The thermal energy absorbed by a sample in a furnace test is directly dependant on the gas phase temperature, but also on the characteristics and conditions within each specific furnace during each test. The thermal properties of the sample being tested will influence the heating of the sample [8], resulting in different heat fluxes being absorbed by every sample. More than half a century of enhancements, essentially in line with additional standardization mechanisms, have been unsuccessful in addressing the fundamental problems with furnace testing [7].

Performance-based structural fire engineering is increasingly common, and is becoming more widely accepted as a method of structural fire resistance design. As a result it is important to better understand the performance of reactive coatings under a range of potential design fire conditions, rather than only under the standard cellulosic case. Alternative methods for performance-based fire engineering design have been explored using heat flux-time curves for determining the heat transfer to structural elements, rather than temperature-time curves. Absorbed heat flux represents a quantification of the flow of thermal energy absorbed into the material and is therefore a more rational metric of thermal loading. Use of heat flux rather than gas temperature as a thermal input (in either testing or modeling) thus avoids many of the problems inherent in furnace testing, and allows fair comparisons between different materials with vastly different thermal properties.

This paper presents a novel testing methodology for studying the performance of reactive coatings when subjected to both standard and non-standard heating regimes. The new approach is calibrated and validated against furnace test data, and it is shown that it offers considerable advantages over conventional testing in terms of reliability, repeatability, versatility, speed and cost. An investigation is then presented to study the effective variable thermal conductivity of a commercially available reactive coating when subjected to various time-histories of heat flux.

\section{MOTIVATION}

Emerging techniques for performance-based structural fire engineering analysis and design require an ability to: (1) define the range of credible worst case design fires for the structure given its geometry, fuel load, physical characteristics, etc; (2) calculate the heat transfer to the structural elements of a building given the design fires selected in (1); and (3) assess the structural response to the resulting heating. Step (2) is problematic for a variety of technical and professional reasons, and as a result the available design codes [9] provide simplified means by which the heat transfer can be conservatively calculated. When reactive coatings are used, this process is complicated by the complex thermo-physical response of the fire protection, making heat transfer calculations to the substrate difficult or impossible [10].

Various attempts have been made to develop computational models which can capture the complexities of swelling, char formation, variable thermal conductivity, etc for reactive coatings in various fire scenarios. For example, Staggs et al. [1] developed a mathematical model to describe the heat transfer and expansion processes of intumescent coatings alongside tests from standard and non-standard furnace tests. The calibrated model was shown to agree well with the experimental results, however, a number of important limitations were observed in the model and it was stated that it is '...difficult to see how a comprehensive description of the expansion process will be achieved and also how it will be translated into a useable mathematical model.' 
Thus, what is needed in practice for any given reactive coating is a simple model which reasonably and conservatively captures the effective thermal conductivity of reactive coatings under various possible heating scenarios. The problem with this approach is that it typically requires numerous costly and time consuming furnace tests of all possible candidate coatings to demonstrate the performance under the potentially infinte range of possible fires; a more versatile and cost-effective testing methodology is needed which can be used for thermal characterization testing.

Recent research on reactive coatings is clearly attempting to move toward performance assessment based on heat flux rather than gas temperature, since this allows for a more accurate and repeatable quantification of the absorbed thermal energy. Several attempts have also been made to address the shortcomings of furnace testing on reactive coatings using cone calorimeters [11, 12, 13, 14]. These studies used cone calorimeter tests to determine thermal properties of the intumescent coating under a range of constant incident heat fluxes. Bench-scale results however are difficult to correlate back to furnace tests, since there is no obvious means by which the imposed (typically presumed constant) incident heat flux of the cone can be correlated to the gas temperatures within a furnace. The variable (reducing) distance between the char surface and the heating elements due to expansion in a cone calorimeter test, and the inability to accurately reproduce established standard heating curves used in furnace, are additional significant problems which must be overcome to support testing of reactive coatings based on heat flux; these are the fundamental problems treated herein.

\section{TEST METHODOLOGY AND PROGRAM}

\section{Novel Heat Transfer Inducing System}

In response to the shortcomings of furnace tests, both in terms of the limitations of the standard fire curve itself in terms of the test approach involving excessive economic and temporal costs, poor repeatability and hence low statistical confidence, a novel test method was developed by Maluk et al. [7] which allows for an accurate quantification of the flow of thermal energy absorbed by a tested element with high precision and good repeatability. The Heat-Transfer Rate Inducing System (H-TRIS) method uses an array of propanefired high performance radiant heaters coupled with a mechanical linear motion system (Fig. 1). The thermal loading of test specimens is controlled using incident heat flux measurements taken by water cooled Schmidt-Boelter heat flux gauges during a pre-programmed calibration procedure. Calibration can be repeated before each new test to account for the specific ambient conditions on any given day, allowing a high level of repeatability between tests [7]. This allows the sample to be thermally loaded following any pre-defined time-history of heat flux by moving the radiant panels towards or away from the sample as necessary. Heat flux curves may thus be programmed for any specific testing furnace and for any target material, using any presumed furnace time-temperature curve. Importantly, all of this can be carried out quickly and repeatability, and costs and order of magnitude less than large scale furnace testing.

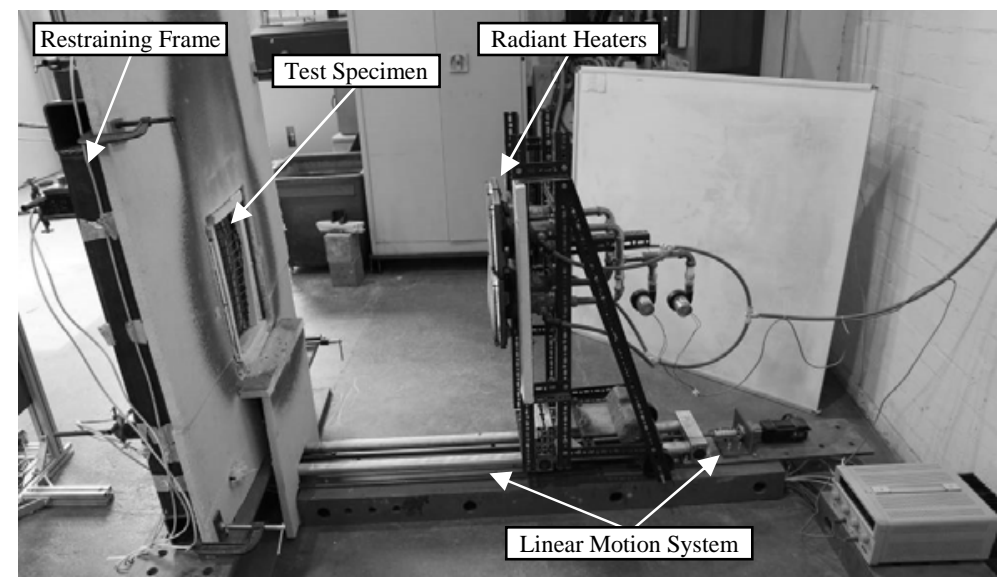

Fig. 1. Photo showing the overall setup and configuration of the H-TRIS testing device (in this configuration being used for spalling tests on concrete). 
The H-TRIS methodology was originally developed to study the propensity of high strength concrete for explosive heat-induced spalling, and it was therefore developed to replicate the thermal energy absorbed by a concrete test specimen during a standard furnace test. The time-history of incident heat flux imposed by H-TRIS, which replicates the thermal conditions experienced by a test specimen in the furnace, was estimated based on results from a numerical inverse heat transfer model developed by Maluk et al. [7]. As input, the model used through-thickness temperature measurements from samples tested in a standard furnace test. Full details of the apporach taken are given by Maluk et al. [7].

A similar method to the one described above cannot be applied to intumescent paints due the complexity of measuring through-thickness temperatures of an expanding char. Also, for the purposes of the current study on reactive coatings it was desired to study coating performance under the standard cellulosic fire, but also under a hydrocarbon exposure and a slow heating 'smouldering' curve [15], when tested in furnaces. In partnership with an industry testing laboratory, a series of furnace tests were performed on unprotected 300 $\mathrm{mm} \times 300 \mathrm{~mm} \times 5 \mathrm{~mm}$ thick unproteceted steel plates (mounted in the furnace walls with their back faces well insulated) under the three aforementioned fire exposures. By measuring the temperature evolution of the steel plate the heat flux absorbed by the steel plate can be calculated for the furnace tests. These data were then input into a fundamentally identical version of the previously developed inverse heat transfer model of H-TRIS' thermal conditions [7] to calculate the incident heat flux required to re-create the thermal response of the unprotected steel samples when subjected to the cellulosic, hydrocarbon, and smouldering curves in the testing furnace; and hence the required position of the radiant array with time with respect to the target samples in order to simulate the furnace tests on unprotected plates in H-TRIS.

Figure 2(a) shows the target gas temperature versus time curves for the standard cellulosic, hydrocarbon, and smouldering fire curves [15], and Fig. 2(b) shows the required incident heat flux versus time curves that required to be imposed by H-TRIS to simulate the respective furnace exposures. H-TRIS was limited in the maximum incident heat flux that could be achieved in its current configuration (Fig. 1), and this is shown by the horizontal lines in Fig. 2(b). It is noteworthy that the consequence of the maximum incident heat flux is that hydrocarbon exposure could only be accurately simulated for about ten minutes, and cellulosic fire exposure for just over thirty minutes.
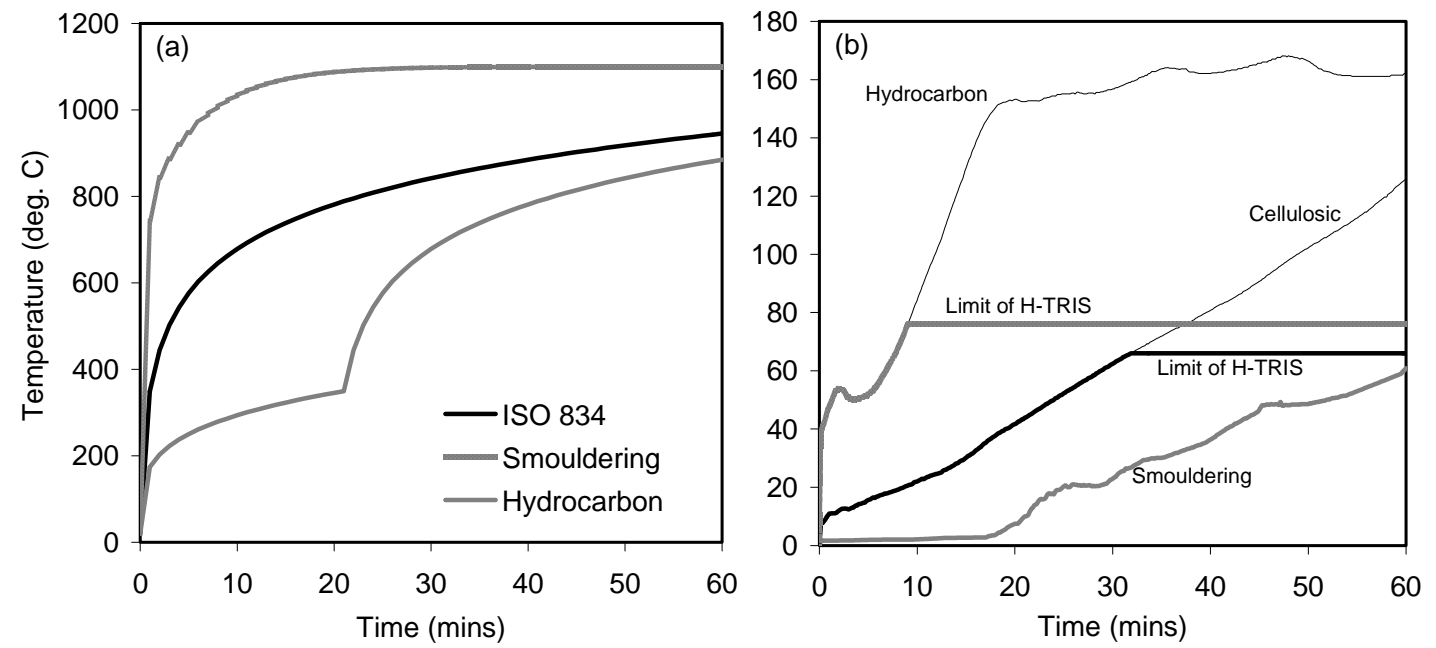

Fig. 2. (a) target gas temperature versus time curves for the standard cellulosic, hydrocarbon, and smouldering fire curves [15], and (b) required incident heat flux versus time curves to be imposed by HTRIS in order to simulate each of these standard fire exposures (note the limits of H-TRIS' maximum incident heat flux in each case). 


\section{Validation}

A series of 'blank plate' validation tests were carried out in H-TRIS in order to verify that the incident heat flux versus time curves shown in Fig. 2(b) accurately reproduced the flow of thermal energy absorbed by unprotected steel plates in furnace tests. Importantly, this forms the basis on which subsequent comparisons were made (see below) between the response of steel plates protected with reactive coatings in furnaces and in H-TRIS. An unprotected steel plate of the same dimensions tested in the furnace was subjected to a calculated required incident heat flux curve for each of the three heating regimes used herein, and the backface temperatures were measured on the steel and compared to those measured during furnace tests on identical plates, thus validating the use of this methodology for reproducing an equivalent absorbed heat flux.

Figure 3 shows a comparison of the average temperatures recorded (with readings taken from five individual thermocouples in each case) at the back of the unprotected steel plates during furnace testing and during H-TRIS testing for samples tested to (a) hydrocarbon, (b) cellulosic, and (c) smouldering fire curves [15]. This figure shows that H-TRIS was able to satisfactorily reproduce the time-history of back-face temperature development in unprotected plates, despite showing a few regions in each case where improvement would be warranted. Figure 3(a) shows that H-TRIS gives lower temperatures beyond about 10 minutes of testing, which is unsurprising given the maximum achievable heat flux limit shown in Fig. 2(b). This suggests that a radiant array with a greater maximum output (or a setup giving smaller losses from the sample surface) will be needed in order to simulate greater durations of hydrocarbon fire exposure. Options for more advanced high-performance radiant heaters are currently being explored. Figure 3(c) shows good agreement between H-TRIS and the furnace test, demonstrating the versatility of this new technique for simulating complex thermal profiles with relative ease.

Figure 3(b), which compares H-TRIS' ability to simulate the cellulosic standard fire, indicates that the heat transfer model of H-TRIS used to predict the theoretical required incident heat flux may require revision. This is most likely due to complexities in determining the appropriate variable view factor for the mobile radiant array, and also to variation in free convective losses from the target surface as the test progresses. An alternative to further refining the inverse heat transfer model would be to manually vary the incident heat flux curve using a trial and error approach. Both approaches are being pursued in ongoing work.

It has been assumed for the purposes of the current paper that the agreement between H-TRIS and the furnace test data is sufficiently accurate to serve the broader objective of studying the response reactive coatings within the range of credible but significantly different heating regimes, such as might be experienced in real fires in real buildings. Testing therefore progressed on the basis of the incident heat flux curves shown in Fig. 2(b).
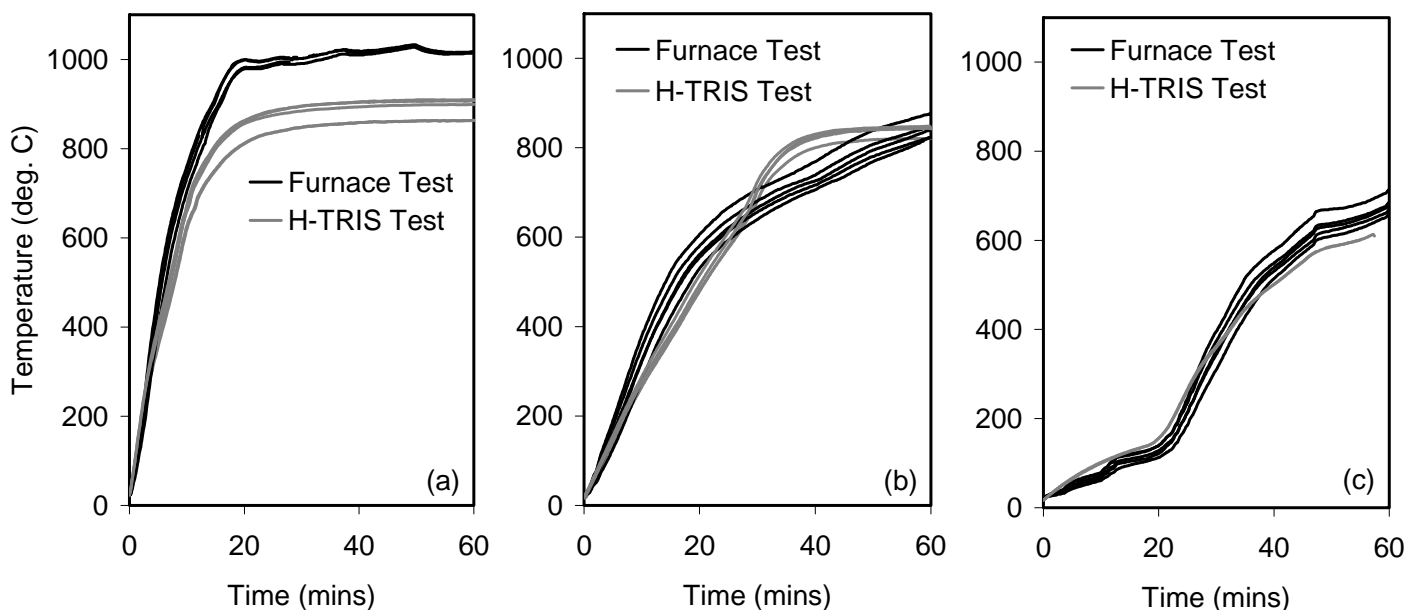

Fig. 3. Back face steel plate temperatures recorded during furnace testing and during H-TRIS testing for unprotected samples tested to (a) hydrocarbon, (b) cellulosic, and (c) smouldering fire curves [15]. Plots show data from five individual thermocouples located as shown in Fig. 4 


\section{Repeatability}

The current predominant means of testing intumescent coatings (e.g. BS EN13381-8 [16]) is based on furnaces whose thermal conditions vary fundamentally between different furnaces due to their size, lining, materials of construction, age, fuel, burner layout, and condition, resulting in variations in the amount of thermal energy absorbed [5, 8]. These factors reduce the validity of comparisons between different furnaces and severely limit the number of repeat tests that can be performed for any given reactive coating. Multiple tests have been carried out using both H-TRIS and in one particular furnace for each heating curve using unprotected steel plates, enabling clear comparisons to be made to investigate the methods' repeatability. The H-TRIS methodology generally exhibited improved homogeneity of temperature across the area of the sample plates, and also excellent repeatability. This is because each test can be preceded by an automatic calibration run which actively measures the incident heat flux emitted from H-TRIS' radiant array with distance from the target surface and feeds this information into the linear motion control system which adjusts its movement accordingly for that specific sample on that specific day. This constant recalibration approach ensures that even different types of radiant panels and test-rig geometries are guaranteed to impose the same time history of incident heat flux, hence enabling data from different H-TRIS style test rigs to be confidently compared with a high degree of accuracy.

\section{Reactive Coatings Testing Program}

The experimental program on reactive coatings consisted of a series of sixteen tests using H-TRIS on protected $300 \mathrm{~mm} \times 300 \mathrm{~mm} \times 5 \mathrm{~mm}$ thick steel plates coated with two different DFTs (2 mm and $4 \mathrm{~mm}$ ) of a commercially available, water-borne intumescent paint which is widely applied in the fire protection of steel framed buildings. To verify the repeatability of testing within H-TRIS, multiple tests were carried out on each paint thickness subjected to each of the three standard fire curves (hydrocarbon, cellulosic, smouldering). Table 1 provides a summary of the tests performed for the current study.

Table 1. Experimental program of H-TRIS tests on steel plates protected with reactive coating.

\begin{tabular}{|l|c|c|}
\hline $\begin{array}{l}\text { Heating Curve } \\
\text { [CEN 1999] }\end{array}$ & DFT (mm) & No. of Tests \\
\hline \multirow{2}{*}{ Cellulosic } & 2 & 3 \\
\cline { 2 - 3 } & 4 & 3 \\
\hline \multirow{2}{*}{ Hydrocarbon } & 2 & 2 \\
\cline { 2 - 3 } & 4 & 2 \\
\hline \multirow{2}{*}{ Smouldering } & 2 & 3 \\
\cline { 2 - 3 } & 4 & 3 \\
\hline
\end{tabular}

Testing was carried out according to the following procedure:

1. The sample was placed inside a sample holding frame, with the sides and back of the steel plate wellinsulated to minimize losses during testing. The back-face of the steel plate was instrumented with five thermocouples, distributed as shown in Fig. 4, before the back-face insulation was applied.

2. The heat exposed face of the protected plate was shielded from the radiant array by a section of ceramic fibreboard, and the radiant panels were lit and allowed to stabilize for 10 minutes.

3. A thermal imaging camera was placed so as to record the surface temperatures of the char during testing. Accurate knowledge of char surface temperature is critical for applying a variable equivalent thermal conductivity model (see below).

4. The fibreboard was removed and H-TRIS' pre-programmed movement was initiated simultaneously.

5. The physical expansion of the reactive coating was assessed at 60 second intervals and the sample holder was manually repositioned (using a graduated pulley system) so as to maintain a constant absolute position of the surface of the intumescent char. This was important in order to be able to ensure that the incident heat flux applied to the surface of the char was the same as assumed when programming H-TRIS. This technique addresses the key shortcoming of testing in a cone calorimeter, 
where the heated surface moves towards the cone during testing and the incident heat flux therefore changes in an unknown way. The sample holder movement was recorded during testing to yield information on the char expansion rates under different heating scenarios for different initial DFTs.

6. Tests were continued for 60 minutes of heating.

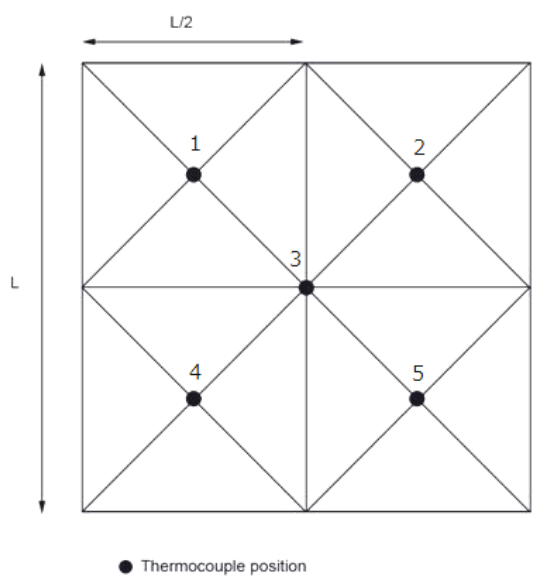

Fig. 4. Back-face thermocouple layout for steel plates during furnace and H-TRIS testing ( $L=300 \mathrm{~mm}$ ).

\section{EXPERIMENTAL RESULTS ON PROTECTIVE COATINGS}

In addition to the problems noted above, testing in furnaces prevents continuous measurement and direct observation of the physical performance of reactive coatings during heating; samples are entirely enclosed in the furnace and small view ports are the only way to observe char formation during heating. Conversely, H-TRIS provides an 'open' test environment and enables detailed observations and measurements to be taken on the coating's physical performance, allowing reactive coating manufacturers access to additional information about the performance of their coatings when subjected to variable heating regimes.

The reactive coating tested in the current study intumesced steadily throughout the heating process, and the expansion was reasonably homogenous across the samples' surface in most cases (Fig. 5). The homogeneity of the expansion varied between the three heating curves. Samples subjected to the hydrocarbon curve were the most homogeneous with a smooth surface texture and even expansion, whereas the cellulosic curve samples were rough and had small pockets of expanded char protruding from the surface. The samples subjected to the smouldering curve exhibited less uniform expansion and in some cases showed evidence of melting and delamination prior to char formation at low heat fluxes; however without any obvious reduction in their thermal insulating performance in terms of their effective variable thermal conductivity (see below).
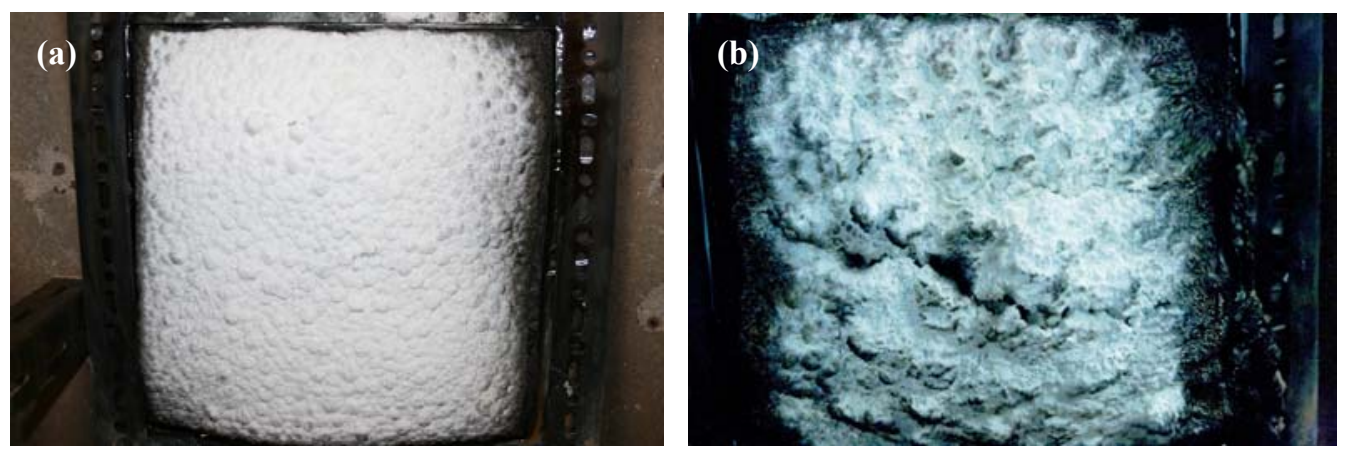

Fig. 5. Char formation under the (a) hydrocarbon and (b) cellulosic incident heat flux exposures when tested in H-TRIS. 
Taken on the whole, the tests performed to date have indicated that the physical performance of the reactive coating tested, assuming that uniform homogenous char is optimal, improves at higher heat fluxes with poorer performance and repeatability being observed under low heat fluxes followed by rapid heating. This is a potentially significant observation, since real fires in real building may be localized, travelling, or may develop more slowly than the standard cellulosic fire. This might therefore be detrimental to the overall performance of some reactive coatings when they have only been certified on the basis of standard furnace tests. Further investigation of this issue is currently underway.

\section{Comparison with Furnace Tests on Protected Plates}

In addition to the tests on protected plates performed using H-TRIS, two tests were also carried out on identical protected plates in a standard fire testing furnaces with DFTs of $2 \mathrm{~mm}$ and $4 \mathrm{~mm}$, respectively, and imposing the cellulosic standard fire [15]. Figure 6 compares the temperatures measured in the protected steel plates for both DFTs under both testing methods. The insulating performance of the paint during the early stages of heating is similar for both DFTs. After approximately 30 minutes however, the back-face temperature measured using H-TRIS increased above those seen in the furnace. By the end of the 60 minute tests this resulted in a $10-20 \%$ temperature difference between the two test methods. It is though that this is primarily due to the previously mentioned overshoot in incident heat flux beyond about 30 minutes (refer to Fig. 3(b)), such that recalibration of H-TRIS' movement is required to better simulate the cellulosic furnace exposure.
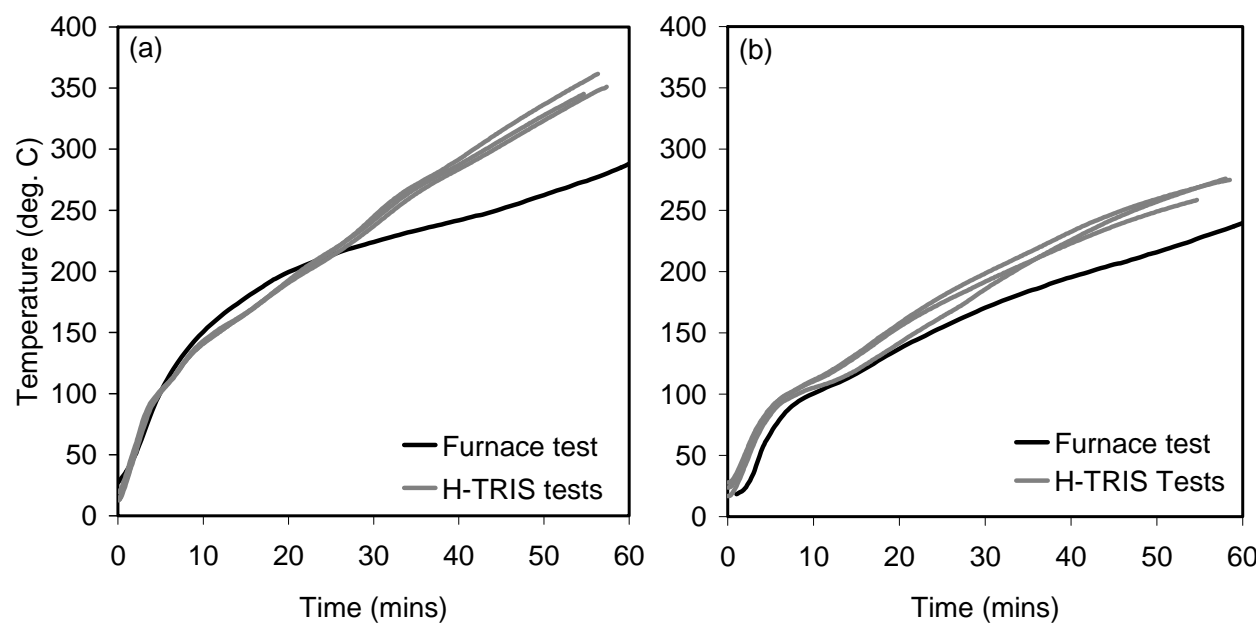

Fig. 6. Average back face temperatures measured on protected steel plates for (a) 2 mm and (b) 4 mm DFTs when tested in the furnace (a single test) and using H-TRIS (three repeat tests) under a cellulosic fire heating regime.

Figure 6 again confirms the excellent repeatability between tests carried out in H-TRIS. While only a total of four tests were carried out using the hydrocarbon curve, these tests showed even better repeatability with temperature differences of less than $5^{\circ} \mathrm{C}$ between respective tests at both DFTs (Fig. 7(a)). Tests performed using the smouldering curve (Fig. 7(b)) showed considerably more variability between tests, as should be expected based on the inferior physical performance of the intumescent coating under this heating regime.

\section{Expansion Rates}

The incident heat flux imposed by H-TRIS is highly sensitive to the distance between the radiant array and the target surface. The heat transfer model and pre-test calibration assume that the target surface remains in a fixed absolute position and only the radiant array moves. To avoid violating this assumption when the coating expands the sample was manually moved away from the radiant array at the same rate as expansion occurred. The added benefit of this method is that it enables measurement of the char expansion rate. This is another novel and significant advantage of testing reactive coatings in H-TRIS as compared with closed furnaces in which char thickness measurements can only be made upon completion of the test. 
It should be noted that char formation was not perfectly homogenous (refer to Fig. 5) and was gauged visually at the thickest location over the target surface. Figure 8 provides char expansion rate data obtained from all 16 tests performed in H-TRIS, and demonstrates both the uniformity and repeatability of response using this technique. This figure also shows, as expected, that the samples exposed to higher heat fluxes expand earlier and to a greater thickness than those exposed to lower heat fluxes; and also that thicker DFTs lead to thicker final char thicknesses, however the benefit of additional DFT is clearly not one-to-one one in terms of final char thickness.
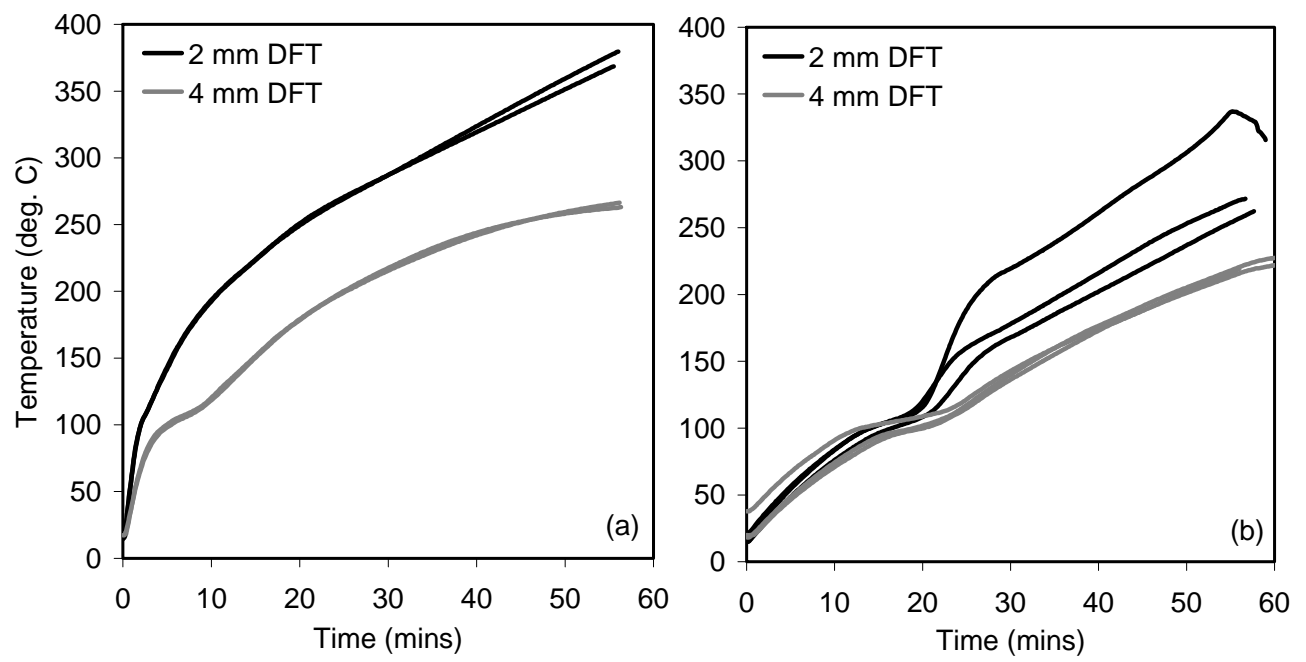

Fig. 7. Average back face temperatures measured in the protected steel plates for $2 \mathrm{~mm}$ and $4 \mathrm{~mm}$ DFTs when tested using H-TRIS under (a) hydrocarbon (two repeat tests) and (b) smouldering fire (three repeat tests) heating regimes.

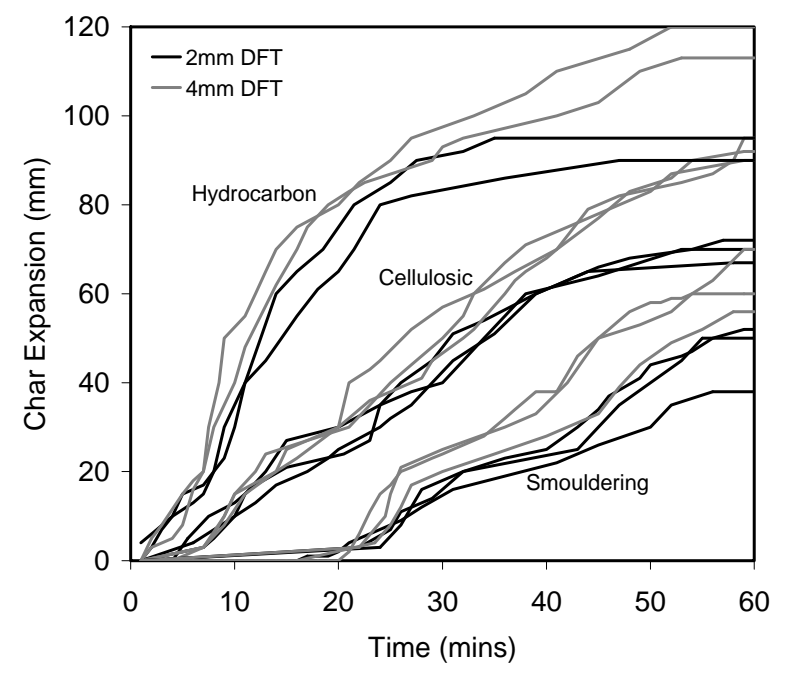

Fig. 8. Expansion of reactive coating versus time of exposure for hydrocarbon (two repeat tests), cellulosic (three repeat tests), and smouldering (three repeat tests) fire curves.

\section{Effective Thermal Conductivity}

The above discussion has shown that H-TRIS can be used as a valid, reliable and versatile method for researchers and reactive coating manufacturers to test their products using non-standard, realistic heating regimes under a variety of credible scenarios. Not only can this be done at lower economic and temporal 
costs, but also with enhanced repeatability as compared with furnace tests. The effective variable thermal conductivity of a given reactive coating can also be carefully characterized using H-TRIS, providing important data which can subsequently be used as inputs for performance-based structural fire design calculations as described below.

\section{Eurocode Variable $\lambda$ Method}

Because the response of reactive coatings to heating is complicated by the various chemical reactions, phase transitions, expansion, and charring phenomena, developing holistic models which account for all relevant parameters to predict their thermal insulating properties under different heating regimes is fraught with complications [1]. As a result, it is typical to treat the thermal conductivity of reactive coatings using an empirically informed procedure called the "Variable $\lambda$ Method" which is set out in Annex E of BS EN 13381-8 [16]. This method is intended for evaluating the thermal conductivity of fire protection systems and is defined by the following difference expression:

$$
\Delta \theta_{a, t}=\frac{\lambda_{p, t} A_{p}}{d_{p} V c_{a} \rho_{a}}\left(\theta_{t}-\theta_{a t}\right) \Delta t
$$

Equation 1, which is simply an energy balance taken during a given time interval during heating, was developed for tests on reactive coatings performed in furnaces, and as such it assumes an adiabatic condition between the furnace gases and the surface char. Whilst this may be a reasonable assumption in furnaces (and there is actually some question as to whether this is the case) it cannot be applied when testing in H-TRIS because the gases surrounding the sample are at ambient temperature and heating is solely by radiation (with losses by natural convection). Because of the above, a thermal imaging technique was used to measure char surface temperature during testing with H-TRIS; in fact, the average char surface temperature over the target surface was taken based on the thermal imaging data.

In any case, Eq. 1 can be rearranged to give the effective thermal conductivity of the reactive coating during any individual time interval during testing for an assumed constant DFT, $d_{p}$. Given that the steel temperature, $\theta_{a, t}$, gas temperature, $\theta_{t}$, and time interval, $\Delta t$, and thermal and physical properties of the steel plate are known during any given time step, the variation in effective thermal conductivity, $\lambda_{p, t}$, can be determined with respect to the gas temperature (as in a furnace test), the char surface temperature (when using H-TRIS), or the steel substrate temperature (perhaps the most useful parameter in practice).

The effective variable thermal conductivity of the reactive coating tested in the current study was calculated for each H-TRIS test using Eq. 1, and the resulting $\lambda_{p, t}$ values are plotted with respect to steel substrate temperature in Figs. 9 and 10. Figure 9 shows the $\lambda_{p, t}$ values for the entire test, providing a detailed view of the pre-expansion effective thermal conductivity, whereas Fig. 10 shows $\lambda_{p, t}$ values on a compressed vertical axis, highlighting the post-expansion effective thermal conductivity.

The agreement between the respective tests and between DFT values is striking, with all coatings tested under all heating regimes giving similar results. It is clear from Fig. 9 that the more severe heat flux of the simulated hydrocarbon fire exposure causes early intumescence as compared with the less severe heat flux of the simulated smouldering fire exposure. However, in every case but one the effective thermal conductivity drops to values of less than about $0.03 \mathrm{~W} / \mathrm{m} \cdot \mathrm{K}$ at a steel substrate temperature of about $110^{\circ} \mathrm{C}$, with remarkable repeatability. The single outlier case was a test in which there was minor delamination of the reactive coating at low temperatures during the early stages of the smouldering fire exposure, causing inferior charring and expansion later in the test. The $\lambda_{p, t}$ values calculated once the char has started expanding are extremely low as a consequence of the assumption that the thickness remains unchanged from the original DFT, which is clearly unphysical however useful for modelling. Nonetheless, the consistency between tests clearly shows that H-TRIS is a powerful and versatile tool for characterization of reactive coatings under a variety of heating regimes and at a variety of DFTs. 


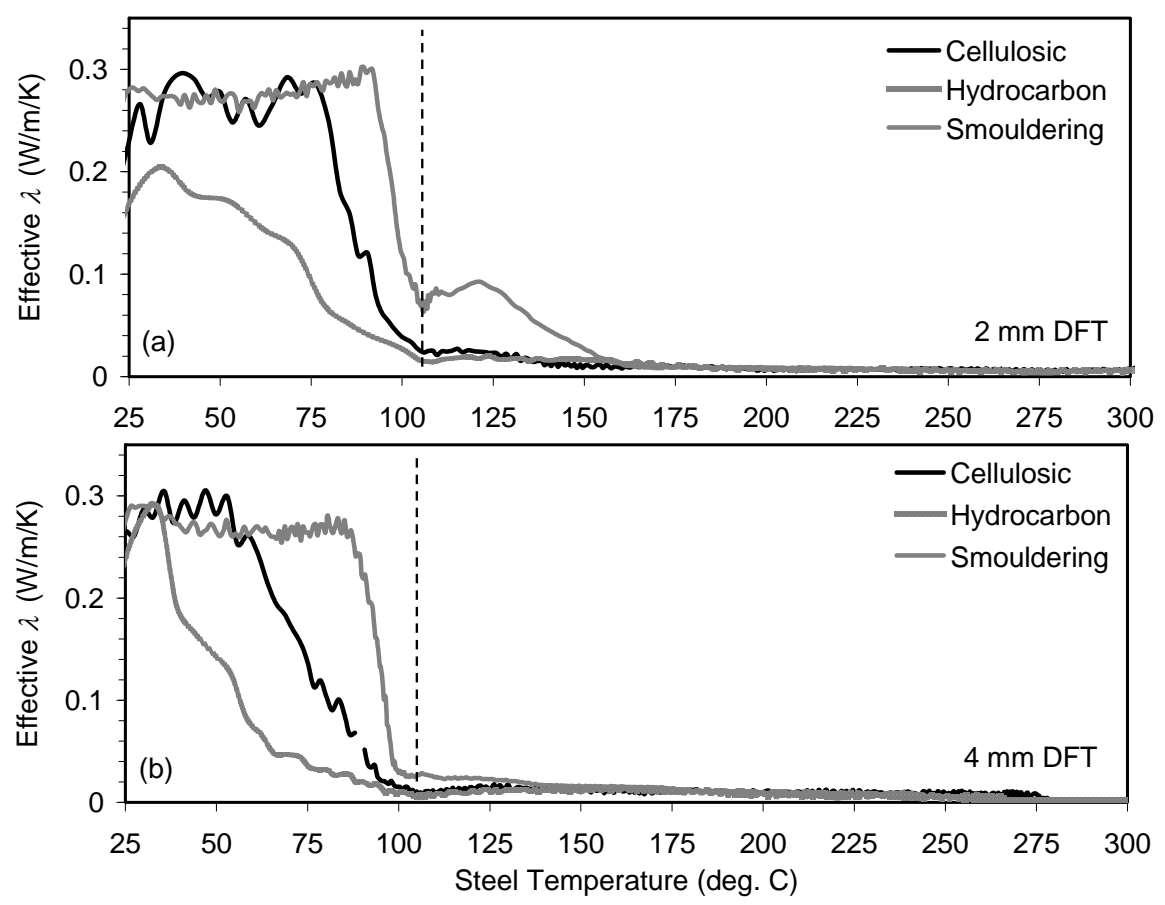

Fig. 9. Selected typical effective variable thermal conductivity, $\lambda_{p, t}$, of reactive coating for H-TRIS tests for (a) $2 \mathrm{~mm}$ DFT and (b) $4 \mathrm{~mm}$ DFT (pre-expansion comparison).
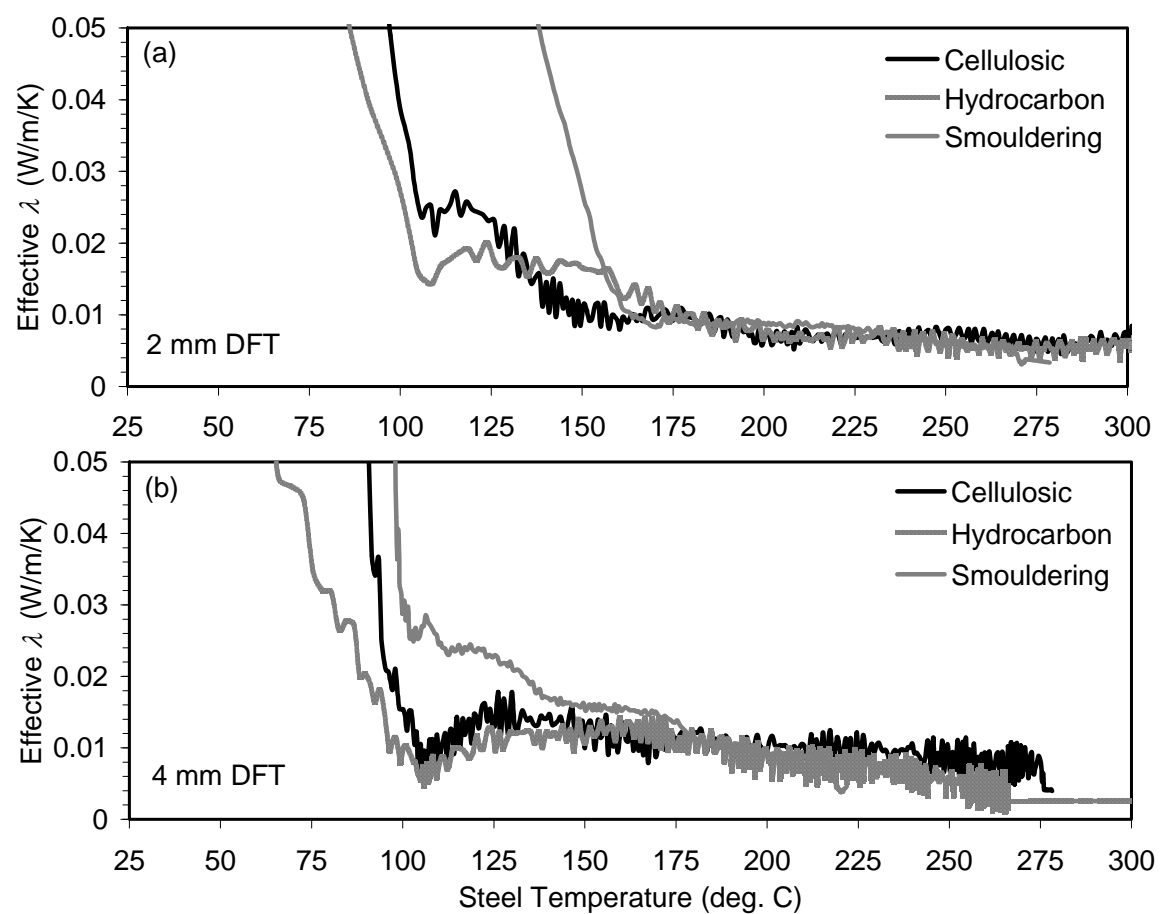

Fig. 10. Selected typical effective variable thermal conductivity, $\lambda_{p, t}$, of reactive coating for H-TRIS tests for (a) $2 \mathrm{~mm}$ DFT and (b) $4 \mathrm{~mm}$ DFT (post-expansion comparison). 


\section{POSSIBLE SUPPORT FOR PERFORMANCE-BASED DESIGN}

To perform rational performance-based structural fire engineering design, engineers need simplified yet rational and conservative thermal models to predict the response of reactive coatings to non-standard heating scenarios. The approach to testing and resulting experimental data presented in the preceding sections may be very useful in this context.

For any given candidate reactive coating, a series of rapid and relatively inexpensive tests could be performed using the H-TRIS testing approach, to provide data similar to those shown in Figs. 9 and 10. These data could then be used to develop a simplified empirical worst case effective variable thermal conductivity model, which could be used as an input in computational heat transfer calculations with any assumed DFT and heating regime (i.e. any design fire). This could potentially be used to define the optimum DFT and to assess the true performance of a protected structure under the resultant heating. The potential advantages of this approach are significant in a performance-based structural design for fire environment, since they could allow structural fire engineers to conservatively quantify the heat transfer to any intumescent protected structure for any presumed design fire scenario. This would potentially allow full fire-structure interaction modelling; currently such analyses are not possible because the requisite data on the performance of reactive coatings are available only for the 'standard' heating curve. The key advantage here is that the H-TRIS testing approach can be used to perform multiple tests under various heat fluxes very quickly and at comparably low cost as compared with furnace tests - thus characterizing the performance of any specific candidate reactive coating under known heating conditions.

Figure 11 shows what such a 'design' effective thermal conductivity curve might look like for the coating used in the tests presented in the current paper. This figure includes all but one of the tests (mentioned above) performed under all heating regimes and for both $2 \mathrm{~mm}$ and $4 \mathrm{~mm}$ DFTs. The 'design' curve shown is simply an example and it should not yet be used in practice. Clearly, a rigorous procedure for defining the 'design' effective thermal conductivity based on the envelope of test data is still needed, and further testing is underway to inform this issue and also to develop practical guidance for use by structural fire engineers.

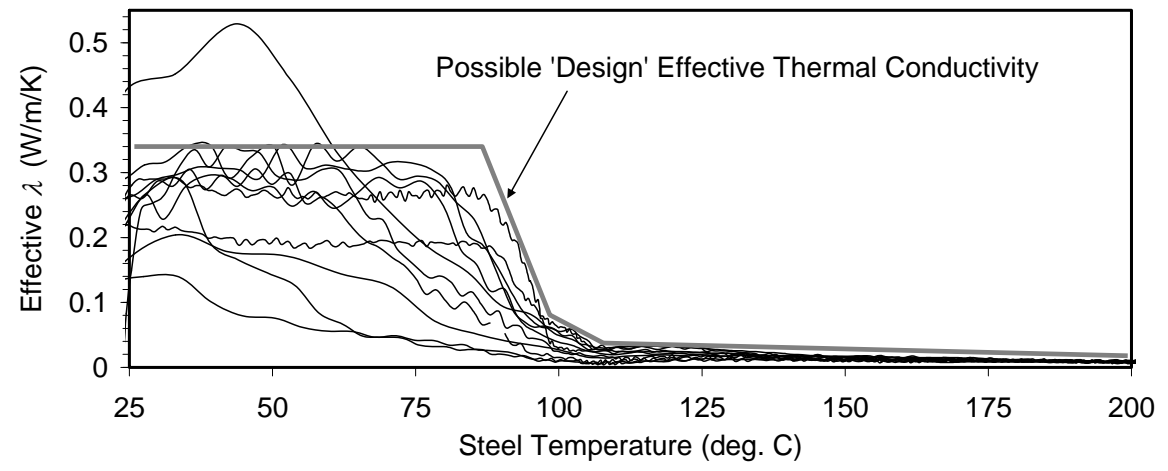

Fig. 11. Illustrative figure conceptually showing one possible determination of 'design' effective variable thermal conductivity, $\lambda_{p, t}$ (grey line) based on a suite of tests performed in H-TRIS (multiple black lines) with varying DFT and severity of heat flux.

\section{CONCLUSIONS}

This paper has presented a novel test methodology for studying the performance of reactive coatings when subjected to non-standard heating regimes. The new approach has been calibrated and validated using furnace test data, and has been shown to offer considerable advantages over conventional furnace testing in terms of reliability, repeatability, versatility, speed and cost. An investigation has been presented studying the performance and effective variable thermal conductivity of a commercially available reactive coating when subjected to various different time-histories of heat flux based on simulations of cellulosic, hydrocarbon, and smouldering furnace test exposures. It has been shown that the heating rate and dry film thickness of the coating do not appear to drastically affect the development of effective thermal conductivity with substrate temperature, leading to a proposal for a simplified method for specifying 
coating requirements and/or performing heat transfer design calculations when designing to non-standard heating regimes. Further work is underway with a view to developing a standardized suite of required tests and a rigorous procedure for definition of the 'design' effective variable thermal conductivity, such that the procedures presented herein can be applied to great advantage for assessment and characterization of any commercially available fire protection coating; intumescent or otherwise.

\section{ACKNOWLEDGEMENTS}

We are grateful for the financial and technical support of International Paint, in particular Dr Allan Jowsey and Mr Ian Bradley for their involvement in this work. We gratefully acknowledge the support of Ove Arup and Partners (Fire Engineering Practice), The Ove Arup Foundation, and The Royal Academy of Engineering. Additional support was provided by the School of Engineering at the University of Edinburgh, which is part of the Edinburgh Research Partnership in Engineering.

\section{REFERENCES}

[1] Staggs, J., Crewe, R., and Butler, R., (2011) A Theoretical and Experimental Investigation of Intumescent Behaviour in Protective Coatings for Structural Steel, Chemical Engineering Science 71: 239-251, http://dx.doi.org/10.1016/j.ces.2011.12.010

[2] CEN, BS EN 1363-1, Fire resistance tests - Part 1: General Requirements, European Committee for Standardization, Brussels, Belgium, 2012, 56 p.

[3] ASTM, E119-12, Standard methods of fire test of building construction and materials, American Society for Testing Materials, West Conshohocken, PA, 2012, 34 p.

[4] Kodur, V., Pakala, P., and Dwaikat, M., (2010) Energy Based Time Equivalent Approach for Evaluating Fire Resistance of Reinforced Concrete beams, Fire Safety Journal 45(4): 211-220, http://dx.doi.org/10.1016/j.firesaf.2010.03.002

[5] Torero, J.L. 2012, “Assessing the True Performance of Structures in Fire,” Proceedings of the $1^{\text {st }}$ International Conference on Performance Based and Life Cycle Structural Engineering, 5-7 December 2012, pp. 429-440.

[6] Parkinson, D., (2007) Performance-Based Design of Structural Steel for Fire Conditions, Steel Structures 7: 219-226.

[7] Maluk, C., Bisby, L., Terrasi, G., Krajcovic M., and Torero J.L., "Novel Fire Testing Methodology: Why, how and what now?," Proceedings of the $1^{\text {st }}$ International Conference on Performance Based and Life Cycle Structural Engineering, 2012, pp. 448-458.

[8] Harmathy, T.Z. and Lie, T.T., "Fire Test Standard in the Light of Fire Research”, Fire Test Performance, ASTM STP 464, American Society for Testing and Materials, 1970, pp. 85-97.

[9] CEN, BS EN 1991-1-2, Actions on structures - Part 1-2: General actions - Actions on structures exposed to fire, Brussels, Belgium, 2002, 62 p.

[10] Anderson, C.E., Ketchum, D.E., and Mountain, W.P., (1988) Thermal Conductivity of Intumescent Chars, Journal of Fire Sciences 6: 390-410, http://dx.doi.org/10.1177/073490418800600602

[11] Bartholmai, M., Schriever, R., and Schartel, B., (2003) Influence of external heat flux and coating thickness on the thermal insulation properties of two different intumescent coatings using cone calorimeter and numerical analysis, Fire and Materials 27: 151-162, http://dx.doi.org/10.1002/fam.823

[12] Griffin, G., (2010) The Modelling of Heat Transfer Across Intumescent Polymer Coatings, Journal of Fire Sciences 5: 249-277, http://dx.doi.org/10.1002/fam.82310.1177/0734904109346396

[13] Zhang, J., Delichatsios, M., McKee, M., \& Ukleja, S., (2011) Experimental and Numerical Study of Burning Behaviors of Flaxboard with Intumescent Coating and nanoparticles in the Cone 
Calorimeter and Single Burning Item Tests. Fire and Materials 36(7): 554-564, http://dx.doi.org/10.1002/fam.1114

[14] Zhang, Y., Wang, Y., Bailey, C., and Taylor, A., (2012) Global Modelling of Fire Protection Performance of Intumescent Coating Under Different Cone Calorimeter Heating Conditions, Fire Safety Journal 50: 51-62, http://dx.doi.org/10.1016/j.firesaf.2012.02.004

[15] CEN, BS EN 1363-2, Fire resistance tests - Part 2: Alternative and additional procedures, European Committee for Standardization, Brussels, Belgium, 1999, 20 p.

[16] CEN, BS EN 13381-8, Test methods for determining the contribution to the fire resistance of structural members - Part 8: Applied reactive protection to steel members, European Committee for Standardization, Brussels, Belgium, 2013, 80 p. 